\title{
Classification of solid renal tumor with oncocytic/eosinophilic cytoplasm: is hybrid oncocytic/chromophobe renal tumor a subtype of oncocytoma, chromophobe renal cell carcinoma, or a distinct tumor entity?
}

\author{
Shuji Mikami ${ }^{1}$, Naoto Kuroda ${ }^{2}$, Yoji Nagashima ${ }^{3}$, Chisato Ohe ${ }^{4}$, Hiroyuki Hayashi ${ }^{5}$ Ryuichi Mizuno ${ }^{6}$, \\ Mototsugu Oya ${ }^{6}$, Kaori Kameyama ${ }^{1}$ \\ ${ }^{1}$ Department of Diagnostic Pathology, Keio University Hospital, Tokyo Japan; ${ }^{2}$ Department of Diagnostic Pathology, Kochi Red Cross Hospital, \\ Kochi, Japan; ${ }^{3}$ Department of Surgical Pathology, Tokyo Women's Medical University, Tokyo, Japan; ${ }^{4}$ Department of Pathology, Kansai Medical \\ University Hospital, Osaka, Japan; ${ }^{5}$ Department of Pathology, Fukuoka University Hospital, Fukuoka, Japan; ${ }^{6}$ Department of Urology, Keio \\ University Hospital, Tokyo, Japan \\ Correspondence to: Shuji Mikami, MD, PhD. Department of Diagnostic Pathology, Keio University Hospital, 35 Shinanomachi, Shinjuku-ku, Tokyo \\ 160-8582, Japan. Email: mikamishuji@gmail.com. \\ Provenance: This is an invited article commissioned by the Section Editor Dr. Xiao Li (Department of Urology, Jiangsu Cancer Hospital, Jiangsu \\ Institute of Cancer Research, Nanjing Medical University Affiliated Cancer Hospital, Nanjing, Chnia). \\ Comment on: Ruiz-Cordero R, Rao P, Li L, et al. Hybrid oncocytic/chromophobe renal tumors are molecularly distinct from oncocytoma and \\ chromophobe renal cell carcinoma. Mod Pathol 2019. [Epub ahead of print].
}

Submitted Sep 01, 2019. Accepted for publication Sep 12, 2019.

doi: $10.21037 /$ atm.2019.09.77

View this article at: http://dx.doi.org/10.21037/atm.2019.09.77

Pathological diagnosis of oncocytic renal tumor is usually difficult task, and their differential diagnosis involves chromophobe renal cell carcinoma (ChRCC), renal oncocytoma (RO), clear cell renal cell carcinoma (CCRCC), epithelioid angiomyolipoma (EAML), etc. Hybrid oncocytic/chromophobe tumor (HOCT) of the kidney is an emerging entity, and there is no description on HOCT in the WHO classification of the urinary system, third edition (1). HOCT was first used to describe tumors showing pathological features of both RO and ChRCC. At the beginning, HOCT has been reported in patients with Birt-Hogg-Dubé syndrome (BHD) $(2,3)$, or those with renal oncocytosis $(4,5)$. Recently, HOCTs have been also reported in patients without background diseases $(6,7)$. Thus, HOCT has been recognized as a renal tumor, but there is only brief description on HOCT in the histopathology section of ChRCC in the 2016 WHO classification of renal tumors as below (8); "A small subset of tumors have overlapping bistology between RO and ChRCC, and which are commonly seen in BHD and renal oncocytosis". It is currently a field of undergoing intense study whether HOCT is a subtype of RO, ChRCC or a distinct renal tumor entity. Therefore, we comment on important studies of HOCT including recently published paper by Ruiz-Cordero et al. (9). In addition, we briefly describe the differential diagnosis of solid renal tumors with oncocytic/eosinophilic cytoplasm from a pathological point of view, which includes oncocytic variant of ChRCC.

Clinically, the median age of the HOCT patients at the diagnosis was seventh and eighth decades, and male patients tended to be slightly more common than female $(7,9,10)$. Characteristic BHD clinicopathological background is observed in HOCTs associated with BHD, but no characteristic symptoms have been reported in HOCTs of non-BHD patients. Many patients with sporadic HOCT had solitary masses, but multiple tumors including bilateral ones occurred in some patients, particularly those with BHD. Majority of HOCT patients seemed to behave indolently without evidence of malignant behavior, but distant metastasis and unclassified renal cell carcinoma (RCC), WHO/ISUP grade 4, arising within background of HOCT were recently reported (9).

Macroscopically, HOCT is well circumscribed and non- 

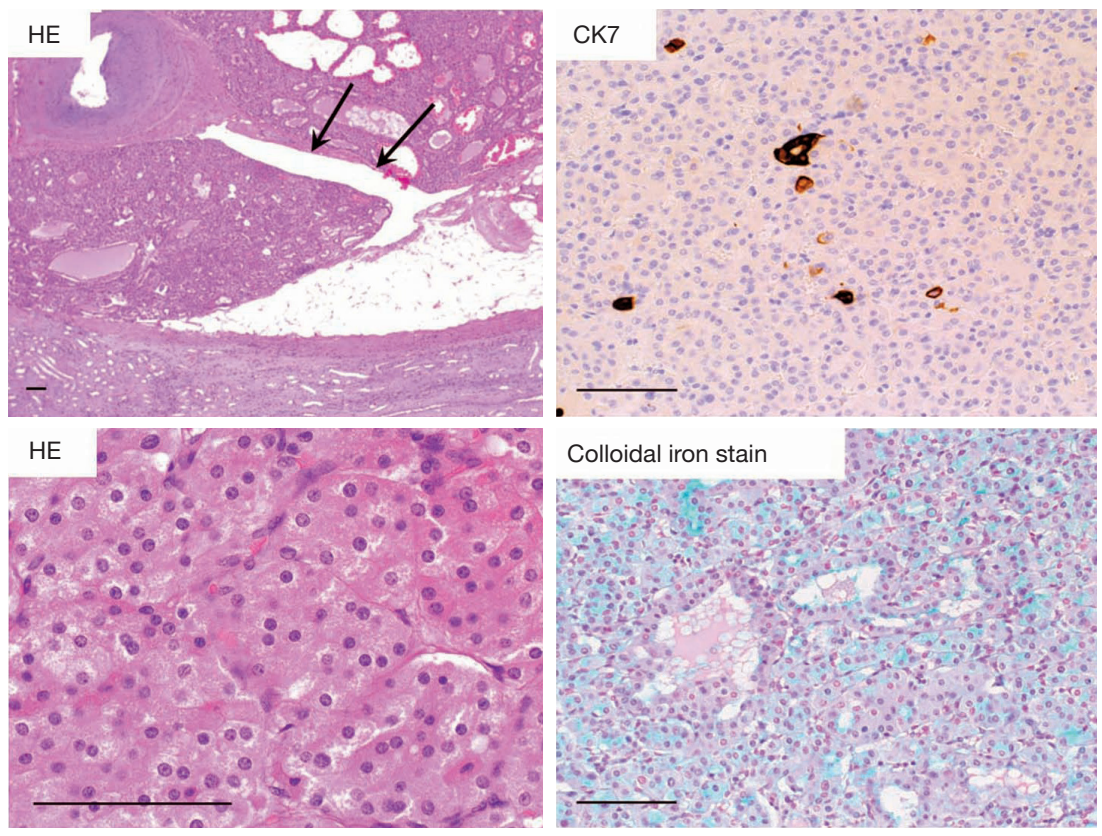

Figure 1 Histopathological features of HOCT. At low magnification, venous invasion was identified (arrows). Oncocytic tumor cells showed perinuclear clearing at high magnification, but no raisinoid nuclei were observed. Focal CK7-positive cells were observed, but most tumor cells were negative for CK7 staining. Therefore, this case was diagnosed as HOCT not ChRCC, eosinophilic variant. Hale's colloidal iron stain was mainly observed in apical portion of the tumor tissue, and focal diffuse staining was also observed. Bars $100 \mu$ m. HOCT, hybrid oncocytic/chromophobe tumor; CK7, cytokeratin 7; ChRCC, chromophobe renal cell carcinoma; HE, haematoxylin and eosin.

encapsulated, and their cut surfaces are homogenous tan to brown without necrosis. Generally, they are localized tumors without invasion to the adipose tissue nor vascular invasion (7). Histologically, HOCTs are consisted of slightly atypical nuclei and rich eosinophilic cytoplasm mainly showing a solid alveolar pattern (Figure 1). Perinuclear halo and binucleated cells are frequently observed (Figure 1), but there are no raisinoid nuclei, characteristic to ChRCC. Perinuclear cytoplasmic clearing is observed in HOCT, resembling ChRCC closely. Tumor cells are similar to those of RO. Some HOCTs might have morphological features both of RO and ChRCC. There might not be a clear boundary between RO-like regions and ChRCC-like regions.

Hale's colloidal iron stain is only focally positive in the apical portion of tumor cells of HOCTs, by contrast to ChRCC with diffuse positive staining. This staining pattern is rather similar to that in RO. Immunohistochemically, all HOCTs are positive for pan-cytokeratin and mitochondrial antigen. Cytokeratin 7 (CK7), E-cadherin, and parvalbumin expression are also observed in most cases, but the proportion of positive cells varies among cases. On the contrary, $\alpha$-methylacyl-
CoA racemase (AMACR), CK20, CD10, carbonic anhydrase 9 (CA9) staining tended to be negative except for focal staining in some tumors. Based on morphological and immunohistochemical characteristics, HOCT is considered to harbor a mixture of the immunohistochemical features that overlap with those of RO and ChRCC.

To elucidate the molecular characteristics of HOCT, analyses were performed by 2 groups, but conflicting results were obtained. Analysis of 14 sporadic HOCTs by fluorescence in situ hybridization and Sanger sequencing revealed that all HOCTs showed some chromosomal imbalances, and the most common numerical aberration was found in chromosome 20 (7). This result is distinct from those of both RO and ChRCC. Another analysis of 10 sporadic HOCTs and 2 HOCTs associated with BHD using array-comparative genomic hybridization showed no chromosome imbalances in about half cases (58\%) (10). These findings and similar Hale's colloidal iron staining pattern suggested HOCTs might be a variant of RO. In recent study by Ruiz-Cordero et al., the mutational and transcriptomic analysis of HOCTs was performed in addition to the DNA copy number analysis (9). This 


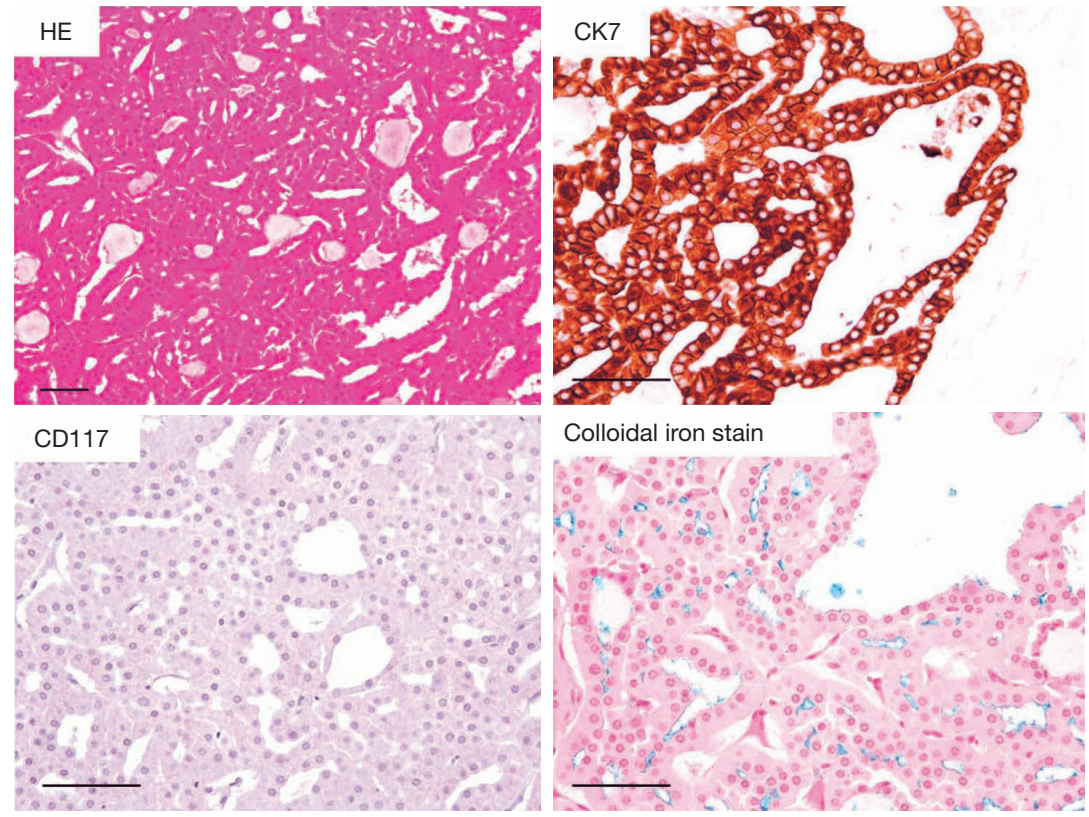

Figure 2 Histopathological features of ChRCC, oncocytic variant. Tumor cells proliferated in predominant tubular pattern with focal solid arrangement. CK7 staining was diffusely positive, but no apparent CD117 staining was observed. Hale's colloidal iron staining was observed in apical portion of tumor cells. Bars $100 \mu \mathrm{m}$. ChRCC, chromophobe renal cell carcinoma; CK7, cytokeratin 7; HE, haematoxylin and eosin.

study revealed that up to $40 \%$ of HOCTs did not show chromosomal arm-spanning imbalances, and the results is consistent with the previous study (10). Losses at chromosome 1 or $\mathrm{X} / \mathrm{Y}$ were predominant copy number aberrations and this is similar to these reported in $\mathrm{RO}$ and eosinophilic variant of ChRCC $(7,10)$. Furthermore, even deep-targeted next-generation sequencing of HOCTs failed to find genetic abnormally in genes reported to be mutated in RO (ERCC2) or ChRCC (TP53 and PTEN). Finally, an analysis of differently expressed genes between RO and ChRCC revealed that HOCTs show an intermediate features between RO and ChRCC, and this results are compatible with the pervious study of three HOCTs associated with BHD (11). These data, altogether, indicates that HOCTs are clinicopathologically and molecular biologically distinct from RO and ChRCC. Therefore, pathologist should render correct diagnosis of HOCT instead of "RO with chromophobe areas" or "tumor of overlapping histology" $(12,13)$.

Correct pathological diagnosis of HOCTs requires accurate differential diagnosis of solid eosinophilic renal tumors, such as CCRCC, ChRCC, RO, EAML, etc. CCRCC with granular or eosinophilic cytoplasm is characterized by rich and fine vascular network and diffuse CA9 staining. A perinuclear halo, raisinoid nuclei, diffuse staining pattern of Hale's colloidal iron stain, CD117, CK7 are observed in ChRCC. Although perinuclear halo is common in HOCTs, no raisinoid nuclei is observed in HOCTs. Typical RO differs is distinct from HOCT in that small tumor cells proliferate in solid alveolar pattern. RO also shows cytoplasmic CD117 staining, but negative or only focally positive for CK7. In addition, Hale's colloidal iron staining was limited to the apical portion of $\mathrm{RO}$ tissue. EAML is sometimes misdiagnosed as high-grade RCC or other tumors. Most importantly, EAML should be suspected in cases where the morphological characteristics of RCC and RO are poor. Immunohistochemically, EAML is negative for CK and EMA, and positive for melanocytic differentiation markers, such as Melan A.

As for differential diagnosis of oncocytic/eosinophilic renal tumor, such as RO, ChRCC and HOCT, a new variant of ChRCC, namely an oncocytic variant of ChRCC, previously proposed by Kuroda et al. should be considered (14). This variant has not been described in the WHO 2016 classification of renal tumors (8), but we diagnose ChRCC, oncocytic variant as often as HOCT in daily pathological diagnosis. Macroscopically, the tumor was well-circumscribed without encapsulation, and the cut surface showed brown without necrosis nor hemorrhage. In ChRCC, oncocytic variant, eosinophilic tumor cells were mainly arranged in tubular pattern, and some cases 
showed focal solid growth (Figure 2). Tumor cells showed relatively uniform in size, indistinct to slightly distinct cell border without apparent perinuclear halo. These findings are similar to those of RO not ChRCC, but diffuse CK7 staining was observed in ChRCC, oncocytic variant, in contrast to negative for focally positive staining in RO. CD117 is negative in ChRCC, oncocytic variant, and this was different from that in RO and typical ChRCC. Furthermore, FISH analysis revealed that the loss of chromosome 7, 10, 13, 17 and 21 in all tumors, which was characteristics of ChRCC. Recently, Trpkov et al. reported low-grade oncocytic tumors of kidney (CD117-negative, CK7-positive), and these tumors were similar to ChRCC, oncocytic variant (15).

In conclusions, these data clearly indicate that HOCT is a distinct tumor entity and that ChRCC, oncocytic variant, is a distinct subtype of ChRCC. Therefore, we think that HOCT should be included in the WHO classification of urinary system, fifth edition, as a new entity, and that the description of ChRCC, oncocytic variant, should be added in histopathology section of ChRCC.

\section{Acknowledgments}

The authors thank Mr. Kazunari Shibata and Takashi Mamizuka, Department of Diagnostic Pathology, Keio University Hospital, for their technical assistance. This manuscript was supported in part by a Grant-in-Aid for Scientific Research (C) (19K07468) from the Ministry of Education, Culture, Sports, Science, and Technology of Japan (MEXT) (Shuji Mikami).

\section{Footnote}

Conflicts of Interest: The authors have no conflicts of interest to declare.

Ethical Statement: The authors are accountable for all aspects of the work in ensuring that questions related to the accuracy or integrity of any part of the work are appropriately investigated and resolved.

\section{References}

1. Eble JN, Sauter G, Epstein JI, et al. editors. World Health Organization of tumours: pathology and genetics of tumours of the urinary system and male genital organs. 3rd ed. Lyon: IARC Press, 2004.
2. Adley BP, Smith ND, Nayar R, et al. Birt-HoggDubé syndrome: clinicopathologic findings and genetic alterations. Arch Pathol Lab Med 2006;130:1865-70.

3. Pavlovich CP, Walther MM, Eyler RA, et al. Renal tumors in the Birt-Hogg-Dubé syndrome. Am J Surg Pathol 2002;26:1542-52.

4. Gobbo S, Eble JN, Delahunt B, et al. Renal cell neoplasms of oncocytosis have distinct morphologic, immunohistochemical, and cytogenetic profiles. Am J Surg Pathol 2010;34:620-6.

5. Tickoo SK, Reuter VE, Amin MB, et al. Renal oncocytosis: a morphologic study of fourteen cases. Am J Surg Pathol 1999;23:1094-101.

6. Mai KT, Dhamanaskar P, Belanger E, et al. Hybrid chromophobe renal cell neoplasm. Pathol Res Pract 2005;201:385-9.

7. Petersson F, Gatalica Z, Grossmann P, et al. Sporadic hybrid oncocytic/chromophobe tumor of the kidney: a clinicopathologic, histomorphologic, immunohistochemical, ultrastructural, and molecular cytogenetic study of 14 cases. Virchows Arch 2010;456:355-65.

8. Moch H, Humphrey P, Ulbright TM, et al. WHO classification of tumours of the urinary system and male genital organs. 4th ed. Lyon: IARC Press, 2016.

9. Ruiz-Cordero R, Rao P, Li L, et al. Hybrid oncocytic/ chromophobe renal tumors are molecularly distinct from oncocytoma and chromophobe renal cell carcinoma. Mod Pathol 2019. [Epub ahead of print].

10. Poté N, Vieillefond A, Couturier J, et al. Hybrid oncocytic/chromophobe renal cell tumours do not display genomic features of chromophobe renal cell carcinomas. Virchows Arch 2013;462:633-8.

11. Klomp JA, Petillo D, Niemi NM, et al. Birt-HoggDubé renal tumors are genetically distinct from other renal neoplasias and are associated with up-regulation of mitochondrial gene expression. BMC Med Genomics 2010;3:59.

12. Kim SS, Choi YD, Jin XM, et al. Immunohistochemical stain for cytokeratin 7, S100A1 and claudin 8 is valuable in differential diagnosis of chromophobe renal cell carcinoma from renal oncocytoma. Histopathology 2009;54:633-5.

13. Trpkov K, Yilmaz A, Uzer D, et al. Renal oncocytoma revisited: a clinicopathological study of 109 cases with emphasis on problematic diagnostic features. Histopathology 2010;57:893-906.

14. Kuroda N, Tanaka A, Yamaguchi T, et al. Chromophobe 
renal cell carcinoma, oncocytic variant: a proposal of a new variant giving a critical diagnostic pitfall in diagnosing renal oncocytic tumors. Med Mol Morphol 2013;46:49-55.

15. Trpkov K, Williamson SR, Gao Y, et al. Low-

Cite this article as: Mikami S, Kuroda N, Nagashima Y, Ohe C, Hayashi H, Mizuno R, Oya M, Kameyama K. Classification of solid renal tumor with oncocytic/eosinophilic cytoplasm: is hybrid oncocytic/chromophobe renal tumor a subtype of oncocytoma, chromophobe renal cell carcinoma, or a distinct tumor entity? Ann Transl Med 2019;7(Suppl 8):S350. doi: 10.21037/atm.2019.09.77 grade oncocytic tumour of kidney (CD117-negative, cytokeratin 7-positive): a distinct entity? Histopathology 2019;75:174-84. 